\title{
Generalized derivations on ideals of prime rings
}

\author{
Emine Albaş
}




\title{
GENERALIZED DERIVATIONS ON IDEALS OF PRIME RINGS
}

\author{
EMINE ALBAŞ
}

Received March 19, 2012

\begin{abstract}
Let $R$ be a prime ring. By a generalized derivation we mean an additive mapping $g: R \rightarrow R$ such that $g(x y)=g(x) y+x d(y)$ for all $x, y \in R$ where $d$ is a derivation of $R$. In the present paper our main goal is to generalize some results concerning derivations of prime rings to generalized derivations of prime rings.
\end{abstract}

2000 Mathematics Subject Classification: 16N60; 16W25; 16R50; $16 \mathrm{U} 80$

Keywords: prime ring, derivation, generalized polynomial, generalized derivation

\section{INTRODUCTION}

Throughout this paper $R$ always denotes an associative prime ring with center $Z(R)$, extended centroid $C$, Martindale quotients ring $Q$ and Utumi quotients ring $U$. For any $x, y \in R$, the commutator of $x$ and $y$ denoted by $[x, y]$ is defined to be $x y-y x$. Recall that a ring $R$ is prime if $x R y=0$ implies $x=0$ or $y=0$. An additive mapping $\alpha: R \rightarrow R$ is called a derivation if $\alpha(x y)=\alpha(x) y+x \alpha(y)$ holds for all $x, y \in R$. The commutativity of prime rings with derivations was initiated by Posner [16]. Over the last two decades, a lot of work has been done on this subject (see $[4,7,11,16]$ where further references can be found). Following Brešar [4], $d: R \rightarrow R$ is called a generalized derivation if there exists a derivation $\alpha$ of $R$ such that

$$
d(x y)=d(x) y+x \alpha(y) \text { for all } x, y \in R .
$$

Hence the concept of generalized derivations covers both the concepts of a derivation and of a left multiplier that is, an additive mapping $f: R \rightarrow R$ satisfying $f(x y)=f(x) y$ for all $x, y \in R$. Basic examples are derivations and generalized inner derivations given by maps of type $f: R \ni x \mapsto a x+x b \in R$ for some $a, b \in R$.

In [9], Hvala initiated generalized derivations from the algebraic viewpoint. In [13], T.K. Lee extended the definition of generalized derivations as follows:

By a generalized derivation we mean an additive mapping $g: I \rightarrow U$ such that $g(x y)=g(x) y+x d(y)$ for all $x, y \in I$, where $I$ is a dense right ideal of $R$ and $d$ is a derivation from $I$ into $U$. 
Moreover Lee also proved that every generalized derivation can be uniquely extended to a generalized derivation of $U$ and thus all generalized derivations of $R$ will be implicitly assumed to be defined on the whole $U$ and obtained the following results:

Theorem 1 ([13], Theorem 3). Every generalized derivation $g$ on a dense right ideal of $R$ can be uniquely extended to $U$ and assumes the form $g(x)=a x+d(x)$ for some $a \in U$ and a derivation $d$ on $U$.

In this paper we extend some well-known results concerning derivations of prime rings to generalized derivations of prime ring.

We note that if $R$ has the property that $R x=0$ implies $x=0$ and $h: R \rightarrow R$ is any function, $d: R \rightarrow R$ is any additive mapping such that $d(x y)=d(x) y+x \alpha(y)$ for all $x, y \in R$, then $d$ is uniquely determined by $h$ and moreover $h$ must be a derivation (see [4], Remark 1).

In all that follows, unless stated otherwise, $R$ will be a prime ring. The related object we need to mention is the two-sided Quotient ring $Q$ of a ring $R$, the twosided Utumi quotient $U$ of a ring $R$ (sometimes, as in [3], $U$ is called the maximal ring of quotients). The definitions, the axiomatic formulations and the properties of these quotient rings $U$ and $Q$ can be found in [2] and [3].

We make a frequent use of the theory of generalized polynomial identities and of the theory of differential identities (see $[3,5,10,12,15]$ ). In particular we need to recall that when $R$ is a prime ring and $I$ a nonzero two-sided ideal of $R$, then $I, R$, $Q$ and $U$ satisfy the same polynomial identities [5] and also the same differential identities [12].

We will also make frequent use of the following result due to Kharchenko [10] (see also [12]):

Let $R$ be a prime ring, $d$ a nonzero derivation of $R$ and $I$ a nonzero two-sided ideal of $R$. Let $f\left(x_{1}, \ldots, x_{n}, d\left(x_{1}\right), \ldots, d\left(x_{n}\right)\right)$ be a differential identity on $I$, that is the relation

$$
f\left(r_{1}, \ldots, r_{n}, d\left(r_{1}\right), \ldots, d\left(r_{n}\right)\right)=0
$$

holds for all $r_{1}, \ldots, r_{n} \in I$.

One of the following holds:

1) Either $d$ is an inner derivation in $Q$, the Martindale quotient ring of $R$, in the sense that there exists $q \in Q$ such that $d(x)=[q, x]$, for all $x \in R$, and $I$ satisfies the generalized polynomial identity

$$
f\left(r_{1}, \ldots, r_{n},\left[q, r_{1}\right], \ldots,\left[q, r_{n}\right]\right) ;
$$

2) or $I$ satisfies the generalized polynomial identity

$$
f\left(x_{1}, \ldots, x_{n}, y_{1}, \ldots, y_{n}\right) \text {. }
$$

In [14], T.K. Lee and W.K. Shiue proved a version of Kharchenko's theorem for generalized derivations and presented some results concerning certain identities with 
generalized derivations. More detail about generalized derivations can be in $[9,13]$ and [14].

We recall some related known result in literature: We say that an additive map $F$ acts as a homomorphism on a nonempty subset $T \subseteq R$, if $F(x y)=F(x) F(y)$ for all $x, y \in T ; F$ acts as an anti-homomorphism on $T$, if $F(x y)=F(y) F(x)$ for all $x, y \in T$; finally $F$ acts as a Jordan homomorphism on $T$ if $F\left(x^{2}\right)=F(x)^{2}$ for all $x, y \in T$. Obviously any additive mapping, which is a homomorphism or an anti-homomorphism, is a Jordan homomorphism. On the other hand, in [8] Herstein proved that in case $R$ is a prime ring of characteristic different from 2, any Jordan homomorphism on $R$ is either a homomorphism or an anti-homomorphism of $R$. In [17], Rehman proved:

Theorem 2 ([17], Theorem 1.2). Let $R$ be a prime ring of characteristic different from 2 and $F$ a nonzero generalized derivation of $R$, with associated derivation $d$. If $F$ acts as homomorphism or anti-homomorphism on a two-sided ideal of $R$, then $R$ is commutative unless $d=0$.

Recently in [6], De Filippis extended the Rehman's result as follows:

Theorem 3 ([6], Theorem 2). Let $R$ be a prime ring, L a noncetral Lie ideal of $R$ and $F$ a nonzero generalized derivation of $R$. If $F$ acts as a Jordan homomorphism on $L$, then either $F(x)=x$ for all $x \in R$, or $\operatorname{char}(R)=2, R$ satisfies the standard identity $s_{4}\left(x_{1}, x_{2}, x_{3}, x_{4}\right), L$ is commutative and $u^{2} \in Z(R)$, for all $u \in L$.

By motivating above results, in the present paper our aim is to obtain a generalization of Rehman's one in [17], moreover this study is a partial generalization of the result in [6] (in case $I=L$ is a two-sided ideal of $R$ ).

Throughout the paper, we denote by $I_{i d}$ the identity map of a ring $R$ (i.e., the map $I_{i d}: R \rightarrow R$ defined by $I_{i d}(x)=x$ for all $\left.x \in R\right)$.

\section{Results}

In the following, we assume that $R$ is a prime ring and that $Z(R)$ is the center of $R$ without stated otherwise.

For the proof of our main results we need the following lemma.

Lemma 1. Let $R$ be a noncommutative prime ring with a generalized derivation $d$ associated with a derivation $\alpha$ of $R$. Suppose that $0 \neq c$ is an element of $R$ such that $c d(x) \in Z(R)$ for all $x \in R$. Then there exists $q \in U$ such that $d(x)=q x$ and $c q=0$.

Proof. By Theorem 1 we can write $d$ as the form $d(x)=q x+\alpha(x)$, where $q \in U$. By the hypothesis we have $c(q x+\alpha(x)) \in Z(R)$ for all $x \in R$. Since $R$ and $U$ satisfy the same differential identity [12] we get

$$
c(q x+\alpha(x)) \in C \quad \text { for all } x \in U
$$


Suppose first that $\alpha \neq 0$. By the result of modulo Kharchenko's Theorem [10] we can divide the proof into two cases.

Assume first that $\alpha$ is an inner derivation of $U$ induced by an element $b \in U$, that is $[b, x]$, for all $x \in U$. In this case $d(x)=q x+[b, x]$. By the hypothesis we have $c(q x+[b, x]) \in C$ for all $x \in U$. Hence above relation implies that

$$
[r, c(q x+[b, x])]=0 \quad \text { for all } r, x \in U
$$

and in particular $c q \in C$. Replacing $x$ by $b$ we get $c q[r, b]=0$ for all $r \in U$. By the primeness of $R$ we obtain that either $c q=0$ or $b \in C$. Since $\alpha \neq 0$ we are forced to consider the first case. Let $c q=0$. By (2.2) we get $[r, c[b, x]]=0$ for all $r, x \in U$. Substituting $x b$ for $x$ in the last relation we have

$$
c[b, x][r, b]=0 \text { for all } r, x \in U .
$$

By the primeness of $U$ and by the supposing on $\alpha$ the above relation implies that $c=0$, a contradiction.

Assume now that $\alpha$ is not an inner derivation of $U$. By Kharchenko's Theorem in [10,12], we get $c(q x+y) \in C$ for all $x, y \in U$. In particular we obtain that $c q x \in C$ for all $x \in U$. Since $R$ is noncommutative prime ring and $c q \in C$ we arrive at $c q=0$. By the last relation we get $c y \in C$ implying that $c=0$, a contradiction.

Thanks to two contradictions we are forced to assume that $\alpha=0$. So we get $d(x)=$ $q x$ and using (2.1) we also obtain that $c q=0$, as asserted.

Now we are ready to prove our main results. The following theorem may be considered as a generalization of [1], Theorem 3.4.

Theorem 4. Let $R$ be a prime ring with center $Z(R)$ and $I$ be a nonzero ideal of $R$. If $R$ admits a nonzero generalized derivation d of $R$, with associated derivation $\alpha$ such that $d(x y)-d(x) d(y) \in Z(R)$ or $d(x y)+d(x) d(y) \in Z(R)$ for all $x, y \in I$, then either $R$ is commutative or $d=I_{i d}$ or $d=-I_{i d}$.

Proof. As we have remarked above we may take a generalized derivation $d$ as the form $d(x)=a x+\alpha(x)$ for all $x \in U$ where $a \in U$ and it is known that $R$ and $I$ satisfy the same differential identity [12]. So we may assume that $R$ admits a generalized derivation such that $d(x y)-d(x) d(y) \in Z(R)$ or $d(x y)+d(x) d(y) \in Z(R)$ for all $x, y \in R$. For each $y \in R$ we consider two subsets $K_{y}=\{x \in R: d(x y)-d(x) d(y) \in$ $Z(R)\}$ and $M_{y}=\{x \in R: d(x y)+d(x) d(y) \in Z(R)\}$. Then $K_{y}$ and $M_{y}$ are two additive subgroups of $(R,+)$ such that $(R,+)=K_{y} \cup M_{y}$; and since a group cannot be the union of two proper subgroups, we have that either $R=K_{y}$ or $R=M_{y}$ for all $y \in R$. Repeating the same above argument we obtain that either $R=\{y \in R: R=$ $\left.K_{y}\right\}$ or $R=\left\{y \in R: R=M_{y}\right\}$. Note that the second case can be reduced to the first case. Indeed, since $f=-d$ is also a generalized derivation of $R$ associated with a derivation $\beta=-\alpha$ the latter case just means that $f(x y)-f(x) f(y) \in Z(R)$ for all $x, y \in R$. Thus we only need to handle the case that

$$
d(x y)-d(x) d(y) \in Z(R) \text { for all } x, y \in R .
$$


If $R$ is commutative we are done. So we may suppose that $R$ is not commutative. For some $a \in U$ write $d(x)=a x+\alpha(x)$ in the last relation. Since $R$ and $U$ satisfy the same differential identity [12] we have

$$
d(x y)-d(x) d(y) \in C \quad \text { for all } x, y \in U .
$$

Take 1 instead of $x$ in (2.3). Hence we get $(1-a) d(y) \in C$ for all $y \in U$.

First suppose that $a \neq 1$. In view of Lemma 1 there exists $q \in U$ such that $d(y)=$ $q y$ for all $y \in U$ and $(1-a) q=0$. By (2.3) we have $q x y-q x q y \in C$ and so $q x(1-q) y \in C$ for all $x, y \in U$. Since $R$ is a noncommutative prime ring the last relation gives us that $q=0$ or $q=1$. The first case implies that $d=0$, a contradiction. Moreover it is easily seen that $a=q$. Thus the second case gives a contradiction.

Now suppose that $a=1$. By (2.3) we have

$$
\alpha(x) \alpha(y) \in C \quad \text { for all } x, y \in U .
$$

Applying Lemma 1 to (2.4), we obtain $\alpha(x) \alpha(y)=0$ for all $x, y \in U$. Replacing $x$ by $x z$ in the last relation we get $\alpha(x) z \alpha(y)=0$ for all $x, y, z \in U$. By the primeness of $U$ we arrive at $\alpha=0$. By the last relation and the assumption $a=1$ we arrive at $d=I_{i d}$, as asserted.

Theorem 5. Let $R$ be a prime ring with center $Z(R)$ and $I$ be a nonzero ideal of $R$. If $R$ admits a nonzero generalized derivation d of $R$, with associated derivation $\alpha$ such that $d(x y)-d(y) d(x) \in Z(R)$ or $d(x y)+d(y) d(x) \in Z(R)$ for all $x, y \in I$, then $R$ is commutative.

Proof. In a similar manner as the proof of Theorem 4 we obtain that either $d(x y)-$ $d(y) d(x) \in Z(R)$ for all $x, y \in R$ or $d(x y)+d(y) d(x) \in Z(R)$ for all $x, y \in R$. As stated before, since the second case can be reduced to the first case by using the observation in the proof of Theorem 4, we consider only the case

$$
d(x y)-d(y) d(x) \in Z(R) \text { for all } x, y \in R .
$$

If $R$ is commutative we are done. So we may suppose that $R$ is not commutative. By Theorem 1, for some $a \in U$ write $d(x)=a x+\alpha(x)$ for all $x \in R$ and since $R$ and $U$ satisfy the same differential identity [12] we have

$$
d(x y)-d(y) d(x) \in C \quad \text { for all } x, y \in U .
$$

Substituting 1 for $y$ in (2.5) we get $(1-a) d(x) \in C$ for all $x \in U$.

If $a \neq 1$, there exits $q \in U$ such that $d(x)=q x$ and $(1-a) q=0$ by Lemma 1 . Using this fact in (2.5) we have

$$
q x y-q y q x \in C \quad \text { for all } x, y \in U .
$$

Replacing $x$ by $x y$ we get $(q x y-q y q x) y \in C$ for all $x, y \in U$. Since $q x y-q y q x \in$ $C$ and $(q x y-q y q x) y \in C$ for all $x, y \in U$, we see that for every $y \in U, q x y-$ $q y q x=0$ for all $x \in U$ or $y \in C$. Recall that $R$ is noncommutative. So $q x y-q y q x=$ 0 for all $x, y \in U$. Setting $x=1$ in the last relation, we get $q U(1-q)=0$. So the 
last relation implies that $q=0$ or $q=1$. If $q=0$, then $d=0$, a contradiction to our hypothesis. If $q=1$, then $x y-y x=0$ for all $x, y \in U$ and hence $R$ is commutative, a contradiction to our assumption.

Now let $a=1$. Then by the hypothesis we have $x y+\alpha(x) y+x \alpha(y)-y x-y \alpha(x)-$ $\alpha(y) x-\alpha(y) \alpha(x) \in C$ for all $x, y \in U$ yielding that

$$
[x, y]+[\alpha(x), y]+[x, \alpha(y)]-\alpha(y) \alpha(x) \in C \quad \text { for all } x, y \in U .
$$

If $\alpha=0$, then (2.6) implies that $[x, y] \in C$ for all $x, y \in U$ which gives us that $R$ is commutative, a contradiction. So we can assume that $\alpha \neq 0$. By Kharchenko's Theorem [10], if $\alpha$ is an inner derivation induced by an element $b \in U \backslash C$ such that $\alpha(x)=[b, x]$ for all $x \in U$ then replacing $y$ by $b$ in (2.6) we get $[x, b]+[\alpha(x), b] \in C$ for all $x \in U$. Taking $x b$ instead of $x$ we have $([x, b]+[\alpha(x), b]) b \in C$ for all $x \in U$. Since $b \notin C$ we obtain $0=[x, b]+[\alpha(x), b]=\alpha(x)+\alpha^{2}(x)$. Replacing $x$ by $\alpha(x)$ in (2.6) and using the last relation we have $\alpha(x) \alpha(y) \in C$. Replacing $y$ by $y b$ in the last relation and using $b \notin C$ we get $\alpha(x) \alpha(y)=0$ for all $x, y \in U$ yielding that $\alpha=0$, a contradiction. If $\alpha$ is not inner, then by Kharchenko's Theorem in [10,12], we get

$$
[x, y]+[z, y]+[x, w]-w z \in C \quad \text { for all } x, y, z, w \in U .
$$

In particular we obtain $[x, y] \in C$ for all $x, y \in U$ yielding that $R$ is commutative, a contradiction.

Example 1. Let $R_{1}$ be any commutative and $R_{2}$ any noncommutative ring. Define the ring $R$ as $R=R_{1} \oplus R_{2}=\left\{(a, b): a \in R_{1}\right.$ and $\left.b \in R_{2}\right\}$. It is clear that $R$ is a noncommutative ring. Let $\delta$ be any derivation of $R_{1}$. Define an additive map $\alpha: R \rightarrow R$ as $\alpha((a, b))=(\delta(a), 0)$, where $(a, b) \in R$. One can be easily shown that $\alpha$ is a derivation on $R$. Then the map $d: R \rightarrow R$ defined as $d((a, b))=(a+\delta(a), b)$ is a generalized derivation on $R$ associated with the derivation $\alpha$. It is easy to verify that $d$ satisfies $d(x y)-d(x) d(y) \in Z(R)$ for all $x, y \in R$, but neither $R$ is commutative, nor $d=0$ nor $d=I_{i d}$.

\section{ACKNOWLEDGEMENTS}

The author would like to express sincere gratitude to the referee for his or her careful reading and making several corrections.

\section{REFERENCES}

[1] E. Albaş and N. Argaç, "Generalized derivations of prime rings," Algebra Colloq., vol. 11, no. 3, pp. 399-410, 2004.

[2] K. I. Beidar, "Rings of quotients of semiprime rings," Vestn. Mosk. Univ., Ser. I, vol. 33, no. 5, pp. 36-43, 1978.

[3] K. I. Beidar, W. S. Martindale, and A. V. Mikhalev, Rings with generalized identities, ser. Pure and Applied Mathematics. New York: Marcel Dekker, 1996.

[4] M. Brešar, "On the distance of the composition of two derivations to the generalized derivations," Glasg. Math. J., vol. 33, no. 1, pp. 89-93, 1991. 
[5] C.-L. Chuang, "GPIs having coefficients in Utumi quotient rings," Proc. Am. Math. Soc., vol. 103, no. 3, pp. 723-728, 1988.

[6] V. De Filippis, "Generalized derivations as Jordan homomorphisms on Lie ideals and right ideals," Acta Math. Sin., Engl. Ser., vol. 25, no. 12, pp. 1965-1974, 2009.

[7] A. Giambruno and I. N. Herstein, "Derivations with nilpotent values," Rend. Circ. Mat. Palermo, II. Ser., vol. 30, pp. 199-206, 1981.

[8] I. N. Herstein, Topics in ring theory, ser. Chicago Lectures in Mathematics. Chicago-London: The University of Chicago Press, 1969.

[9] B. Hvala, "Generalized derivations in rings," Commun. Algebra, vol. 26, no. 4, pp. 1147-1166, 1998.

[10] V. K. Kharchenko, "Differential identities of prime rings," Algebra Logic, vol. 17, pp. 155-168, 1978.

[11] P. H. Lee and T. K. Lee, “On derivations of prime rings," Chin. J. Math., vol. 9, no. 2, pp. 107-110, 1981.

[12] T.-K. Lee, "Semiprime rings with differential identities," Bull. Inst. Math., Acad. Sin., vol. 20, no. 1, pp. 27-38, 1992.

[13] T.-K. Lee, "Generalized derivations of left faithful rings," Commun. Algebra, vol. 27, no. 8, pp. 4057-4073, 1999.

[14] T.-K. Lee and W.-K. Shiue, "Identities with generalized derivations," Commun. Algebra, vol. 29, no. 10, pp. 4437-4450, 2001.

[15] W. S. Martindale, "Prime rings satisfying a generalized polynomial identity," J. Algebra, vol. 12, pp. 576-584, 1969.

[16] E. C. Posner, "Derivations in prime rings," Proc. Am. Math. Soc., vol. 8, pp. 1093-1100, 1957.

[17] N. ur Rehman, "On generalized derivations as homomorphisms and anti-homomorphisms," Glas. Mat., III. Ser., vol. 39, no. 1, pp. 27-30, 2004.

\section{Author's address}

\section{Emine Albaş}

Ege University, Science Faculty, Department of Mathematics, 35100 Bornova, Izmir, Turkey

E-mail address: emine.albas@ege.edu.tr 